\title{
Identifying sputum specimens of high priority for examination by enhanced mycobacterial detection, identification, and susceptibility systems (EMDISS) to promote the rapid diagnosis of infectious pulmonary tuberculosis
}

\author{
R Freeman, J Magee, A Barrett
}

\begin{abstract}
Aims-To compare clinical information and sputum microscopy as methods for the selection of samples for enhanced mycobacterial detection, identification, and susceptibility systems (EMDISS) to promote the rapid diagnosis of patients with infectious pulmonary tuberculosis. Methods-Two thousand, two hundred and sixty four specimen request forms were examined for clinical details, which were then used to identify specimens likely to yield Mycobacterium tuberculosis on culture. These results were compared with the results of sputum microscopy for acid fast bacilli (AFB). Both methods were assessed against the results of culture using a combination of continuous automated mycobacterial liquid culture (CAMLiC) and conventional solid culture. Results-Classification based on clinical details was an inefficient method of identifying high priority specimens for EMDISS. Although, when given, clinical details were often consistent, a substantial proportion of specimens arrived with no details. This approach would result in the referral of at least $16 \%$ of the workload but lead to the detection by culture of only $46 \%$ of the $M$ tuberculosis present within it. In contrast, microscopy for AFB defined a much smaller number of specimens $(4.8 \%$ of the total), which contained $\mathbf{9 0 \%}$ of the $M$ tuberculosis isolates.

Conclusions-Microscopy for AFB is the most efficient method for defining sputum specimens suitable for referral for enhanced mycobacteriological techniques. However, it is essential that the methods used for smear preparation and microscopy are of the highest possible standard, otherwise some patients with infectious pulmonary tuberculosis will be denied, unnecessarily, the benefits of important advances in mycobacteriology.

(f Clin Pathol 2001;54:613-616)
\end{abstract}

Keywords: tuberculosis; diagnosis; AFB smear; mycobacterial culture

New laboratory methods, including continuous automated mycobacterial liquid culture (CAMLiC), ${ }^{1}$ molecular techniques such as species specific gene probes for identification ${ }^{2}$ and Mycobacterium tuberculosis specific polymerase chain reaction (PCR) methods for use on sputum, ${ }^{3}$ permit suitably equipped laboratories to meet the standards defined by the Centers for Disease Control and Prevention (the "CDC criteria") for a modern mycobacteriology service. ${ }^{4}$ These criteria specify that the detection and identification of $M$ tuberculosis and the determination of susceptibilities to rifampicin, isoniazid, ethambutol, and pyrazinamide should be completed within 30 days of the receipt of the specimen. Such methods not only enhance greatly the speed with which these tasks can be accomplished but, particularly as a result of CAMLiC, also significantly increase the yield of mycobacteria, including $M$ tuberculosis, from the sputum samples processed in this way. The more efficient enhanced mycobacterial detection, identification, and susceptibility testing service (EMDISS), which results, permits earlier diagnosis and treatment of tuberculosis, facilitates contact tracing, and allows early identification of resistant strains of $M$ tuberculosis.

If all mycobacteriology specimens are to be examined by these modern methods there will be substantial costs for equipment, additional sums to meet the more stringent safety and containment standards for mycobacterial liquid culture, and a probable reorganisation of the existing laboratory systems. In the UK, for instance, nearly 400000 mycobacterial specimens underwent primary processing by 257 laboratories in $1995 .^{5}$ This workload could be processed to the new standard by a much smaller number of properly equipped specialist mycobacterial centres, thereby achieving considerable economies of scale in both equipment and costs, and also concentrating the technical expertise demanded by the new methods. Such developments will take some time. However, as a first measure, it has been proposed that high priority sputum samples from those patients most likely to have infectious pulmonary tuberculosis be referred to laboratories already operating EMDISS, the small delay incurred by transport being more than offset by the much reduced turnaround time achieved by referral. ${ }^{67}$ Therefore, it is important to develop criteria for the selection of such high priority specimens.

To date, the only criterion used has been the microscopical detection of acid fast bacilli (AFB) in the sputum specimen. However, the 
sensitivity of this technique ("the smear") is reported to vary from $60 \%$ to $80 \%$ when assessed against culture ${ }^{8}$ suggesting that some specimens from patients with infectious pulmonary tuberculosis will not be identified. We have explored an alternative approach for the identification of high priority sputum specimens; using the clinical details supplied on the laboratory request form as a means of categorising specimens, we compared these results with assessments derived from smear examinations, and then compared both sets of assessments with the results of culture by a combination of CAMLiC and conventional culture (the basis of EMDISS).

In addition to its role as a reference centre for mycobacteria, Newcastle Public Health Laboratory provides a primary diagnostic mycobacteriology service for several local hospitals and surrounding districts and this specimen flow was used for the study.

\section{Methods and materials}

SPECIMEN REQUEST FORMS AND SPECIMEN RECORDS

For the same 10 week period (1 August to 14 November) in each of the three years 1997 1999 all laboratory request forms from sputum specimens undergoing EMDISS were inspected. Specific clinical and radiographic details were sought and specimens were then categorised as follows:

(1) High priority: previous history of tuberculosis, night sweats, or haemoptysis; $x$ ray appearances included upper lobe disease or cavitation.

(2) Medium priority: signs and symptoms of pneumonia or pleurisy or productive cough; $x$ ray appearances included pneumonia (but not specified as upper lobes), hilar lymphadenopathy, or pleural effusion.

(3) Low priority: signs and symptoms were non-specific-for example, fever, respiratory infection, or cough; $x$ ray appearances not stated.

(4) Unclassifiable: no clinical details supplied.

Request forms for 2264 sputum specimens from 1242 patients were examined and categorised. A note was made of the number of specimens for each patient and the consistency of the clinical details (where provided) in successive specimens from the same patient. Specimens from patients known to be undergoing treatment for recently diagnosed tuberculosis or another mycobacteriosis were excluded from this part of the study. The results of microscopy for AFB and of combined CAM$\mathrm{LiC}$ and conventional culture were recorded for these specimens.

Finally, the results for all sputum specimens (that is, not simply those in the selected 10 week period) in the years 1997, 1998, and 1999 undergoing microscopy and culture by both CAMLiC and parallel conventional solid culture were retrieved; they comprised 1329, 1744 , and 2860 specimens, respectively. A note was made of the smear and culture results for all these specimens. A positive culture was defined as the recovery of a mycobacterium in either culture system. These data were used to calculate the sensitivity, specificity, positive predictive value (PPV), and negative predictive value (NPV) for the smear relative to CAMLiC plus conventional culture over the entire three year period. The results on specimens taken from patients known to be receiving antimycobacterial treatment were included in this part of the study.

\section{SPECIMEN PROCESSING}

Sputum was digested using an equal volume of dithiothreitol (Sputasol ${ }^{\mathrm{TM}}$ ) and vortex mixed until homogeneous. The homogenate was centrifuged for 15 minutes at $3000 \times g$ and the supernatant was discarded. A thin smear was made from the deposit, which was heat fixed and stained by the auramine-phenol method, then examined under UV light for the presence of AFB. The remainder of the deposit was treated with $4 \% \mathrm{wt} / \mathrm{vol} \mathrm{NaOH}$ for 25 minutes, with regular vortex mixing at five minute intervals, and then neutralised with $\mathrm{N} / 2 \mathrm{H}_{2} \mathrm{SO}_{4}$ and recentrifuged. The concentrated deposit was used to inoculate Loewenstein-Jensen (LJ) slopes and $\mathrm{MB} / \mathrm{BacT}$ bottles. LJ slopes were inspected weekly for eight weeks and the $\mathrm{MB} / \mathrm{BacT}$ bottles were processed for 28 days on the CAMLiC system. Mycobacteria detected in either culture system were identified as $M$ tuberculosis using a gene probe method (Gene-Probe Inc, San Diego, California, USA) or, when not found to be $M$ tuberculosis, as other mycobacterial species by a combination of gene probes and phenotypic tests.

\section{Results}

Table 1 shows the associations between the clinically based categorisation of the specimens using details supplied on the request form. It can be seen that 58 of the 141 mycobacteria isolated $(41 \%)$ and 32 of the 70 isolates of $M$ tuberculosis (46\%) were recovered from those specimens designated as high priority by this means. Similarly, 63\% (89 of 141) of the positive mycobacterial cultures and $73 \%$ (51 of 70 ) of the $M$ tuberculosis isolates were from specimens designated as high or medium priority. However, 15 mycobacteria, including 10 that were $M$ tuberculosis, were isolated from 521 specimens from patients in whom the clinical details were non-specific and 37 mycobacteria, including nine examples of $M$ tuberculosis, were isolated from 823 specimens with no accompanying clinical information at all. Table 1 also shows that the smear was positive in 63 of 70 specimens that yielded $M$ tuberculosis on culture $(90 \%)$ compared with only 46 of 71 specimens found to contain non-tuberculous mycobacteria $(64.8 \%)$. This difference is significant $\left(\chi^{2}=11.4 ; \mathrm{p}<0.001\right)$.

Three or more specimens were received from 345 of the 1242 patients (28\%) whose specimen request forms were assessed. At least two specimens were received from 562 (45\%) of these patients. The clinical details on the request forms were consistent (resulted in the same categorisation) in 425 of the 562 instances $(76 \%)$ in which more than one specimen was received from the same patient, but in 
Table 1 Results of the categorisation of 2264 sputum specimens from 1242 patients for their likelihood to contain Mycobacterium tuberculosis based on clinical details supplied on request forms compared with the results of sputum microscopy for acid fast bacilli and the results of culture

\begin{tabular}{|c|c|c|c|c|c|}
\hline & \multicolumn{4}{|c|}{ Categories (see text for details) } & \multirow[b]{2}{*}{ Total } \\
\hline & Unclassified & Low priority & Medium priority & High priority & \\
\hline Number of specimens & 823 & 521 & 561 & 359 & 2264 \\
\hline Isolates of all mycobacteria (smear +ve samples) & $37(23)$ & $15(11)$ & $31(24)$ & $58(50)$ & $141(100)$ \\
\hline Isolates of $M$ tuberculosis (smear +ve samples) & $9(9)$ & $10(8)$ & $19(17)$ & $32(29)$ & $70(63)$ \\
\hline$\%$ Isolation of $M$ tuberculosis & 1.1 & 1.9 & 3.4 & 8.9 & 3.1 \\
\hline
\end{tabular}

$33 \%$ of these (140 of 425 ) the consistency simply reflected the fact that no clinical details were given in successive requests.

Review of the smear results relative to combined CAMLiC and LJ culture for the 1329, 1744 , and 2860 sputum specimens processed in 1997, 1998, and 1999 showed that the sensitivity was $0.81,0.82$, and 0.77 , respectively, for the three years. Specificity was shown to be $0.99,0.99$, and 0.99, respectively. PPV was $0.94,0.92$, and 0.9 and NPV was $0.97,0.98$, and 0.98, respectively. In 1997, nine smear positive/culture negative samples occurred and seven of these were from patients on antimycobacterial treatment at the time. In 1998, 11 smear positive/culture negative specimens occurred, eight of which were from patients undergoing treatment. In 1999, 19 such specimens occurred and concomitant antimycobacterial treatment occurred in 16 . Over the three year period, involving an assessment based on all 5933 specimens, the sensitivity, specificity, PPV, and NPV of the smears was 0.8, 0.99, 0.92 , and 0.98 , respectively. Therefore, "false positive smears" totalled 3931, 79\% of which were from specimens taken during antimycobacterial treatment. These figures refer to isolates of all mycobacterial species and are not specific to $M$ tuberculosis.

These results show that sputum microscopy for AFB is the most efficient method for the identification of high priority specimens for referral for EMDISS. Using the smear as the only criterion, a calculated 109 specimens (number of positive smears in the analysis of the request forms $(100) \times$ the reciprocal of the PPV for the AFB smear technique (0.92), thereby allowing for the predicted number of "false positives") would have been referred, resulting in the isolation of 100 mycobacteria, including 63 of $70(90 \%)$ of the isolates of $M$ tuberculosis. These specimens comprised $4.8 \%$ of the total workload. Only 41 specimens that contained mycobacteria on culture would not have been referred, including seven that contained $M$ tuberculosis.

Conversely, if referral was based on the clinical assessments, 359 high priority specimens would have been referred $(15.8 \%$ of the workload), resulting in the recovery of 58 mycobacteria, 32 of which were $M$ tuberculosis; an efficiency of only $45.7 \%$ for the detection of culture positive pulmonary tuberculosis. The additional referral of medium priority specimens would have resulted in 920 specimens going on for EMDISS, but still only permitting the isolation of 51 of $70 \mathrm{M}$ tuberculosis $(72.8 \%)$. These specimens comprised $40.6 \%$ of the workload. Combining the two approaches by referring all clinical high priority specimens (359) plus all smear positive specimens in the other categories (58) would have resulted in the recovery of 66 of 70 isolates of $M$ tuberculosis; a gain of only three isolates for the burden of the additional 288 specimens when compared with the referral of only smear positive sputum.

\section{Discussion}

Attempts to assess the priority of sputum specimens for enhanced mycobacterial analysis by patient characteristics are inevitably constrained by the amount and detail of the clinical information given on the request form. Our study made the assessment using only the information available at receipt of the specimen and therefore concentrated on those patient details usually supplied on request forms. Successful clinical predictive models have been reported $^{9-11}$ that utilise such data as ethnicity, immigration status, underlying disease (for example, alcoholism), and human immunodeficiency virus status in addition to the symptoms and radiological features used here. Nonetheless, the characteristics used in our study (haemoptysis, night sweats, previous tuberculosis, upper lobe disease, and cavitation) were all shown, inter alia, to be strongly predictive of active tuberculosis in those models. The modified approach used in our study resulted in a sensitivity (for high priority specimens) of only $45.7 \%$ compared with a sensitivity of $79.5 \%$ for the smear. It is particularly regrettable that no clinical details were supplied for $36.3 \%$ of the specimens, especially when 37 mycobacteria, including nine isolates of $M$ tuberculosis, were recovered from this category. Informal enquiries revealed that clinical details that would have placed some of these specimens in the high priority group could have been made available. Thus, although the clinical classification produced a high priority group in which the presence of $M$ tuberculosis by culture was increased almost threefold compared with the whole specimen workload $(8.9 \%$ v $3.1 \%)$, the smear results defined a specimen group in which the yield of $M$ tuberculosis increased almost 30 fold.

Our results suggest that referral of smear positive sputum specimens to suitably equipped and experienced laboratories for EMDISS is an extremely efficient and cost effective means of providing to almost all patients with infectious pulmonary tuberculosis the speed and sensitivity in diagnosis and susceptibility studies that modern mycobacteriology offers, provided, always, that the microscopy method used is of a sufficiently high standard for the purpose. ${ }^{8}$ 
1 Magee JG, Freeman R, Barrett A. Enhanced speed and sensitivity in the cultural diagnosis of pulmonary tuberculosis a continuous automated mycobacterial liquid culture Peterson EM,

2 Peterson EM, Lu R, Floyd C, et al. Direct identification of Mycobacterium tuberculosis, $M$. avium and $M$. intracellulare from amplification of primary cultures in BACTEC media, using DNA probes. F Clin Microbiol 1989;27:1543-7.

3 Kearns AM, Freeman R, Steward M, et al. A rapid polymerase chain reaction technique for the detection of Mycobacterium tuberculosis in a variety of clinical specimens. $\mathcal{F}$ Clin Pathol 1998;51:922-4.

4 Tenover FC, Crawford JC, Huebner RE, et al. The resurgence of tuberculosis: is your laboratory ready? $7 \mathrm{Clin}$ Microbiol 1993;34:680-5.

5 Drobniewski FA, Watt B, Smith EG, et al. A national audit of the laboratory diagnosis of tuberculosis and othe

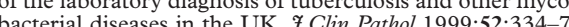

6 Salfinger M. Diagnosis of tuberculosis and other diseases caused by mycobacteria. Infection 1997;25:60-2.

7 Salfinger M, Pfyffer GE. The new diagnostic mycobacteriology laboratory. Eur f Clin Microbiol Infect Dis 1994;13:96179.

8 American Thoracic Society. Diagnostic standards and classification of tuberculosis in adults and children. $A m \mathcal{F}$ Respir Crit Care Med 2000;161:1376-95.

9 El-Sohl A, Mylotte J, Sherif S, et al. Validity of a decision tree for predicting active pulmonary tuberculosis. Am $\mathcal{F}$ Respir Crit Care Med 1997;155:1711-16.

10 El-Sohl A, Hsiao C-B, Goodnough S, et al. Predicting active pulmonary tuberculosis using an artificial neural network. Chest 1999;116:968-73.

11 Cohen R, Muzaffar S, Capellan J, et al. The validity of classic symptoms and chest radiographic configuration in predicting pulmonary tuberculosis. Chest 1996;109:420-3.

\section{Narrative Based Medicine, An Interdisciplinary Conference}

Research, Narrative, and Practice

A two day conference-Monday 3rd and Tuesday 4th September 2001

Homerton College, Cambridge, UK

BMF Publishing Group

For full details contact: BMA/BMJ Conference Unit, Tavistock Square, London, WC1H 9JP Tel: +44 (0)20 7383 6819; fax: +44 (0)207383 6663; email: clyders@bma.org.uk.

www.quality.bmjpg.com

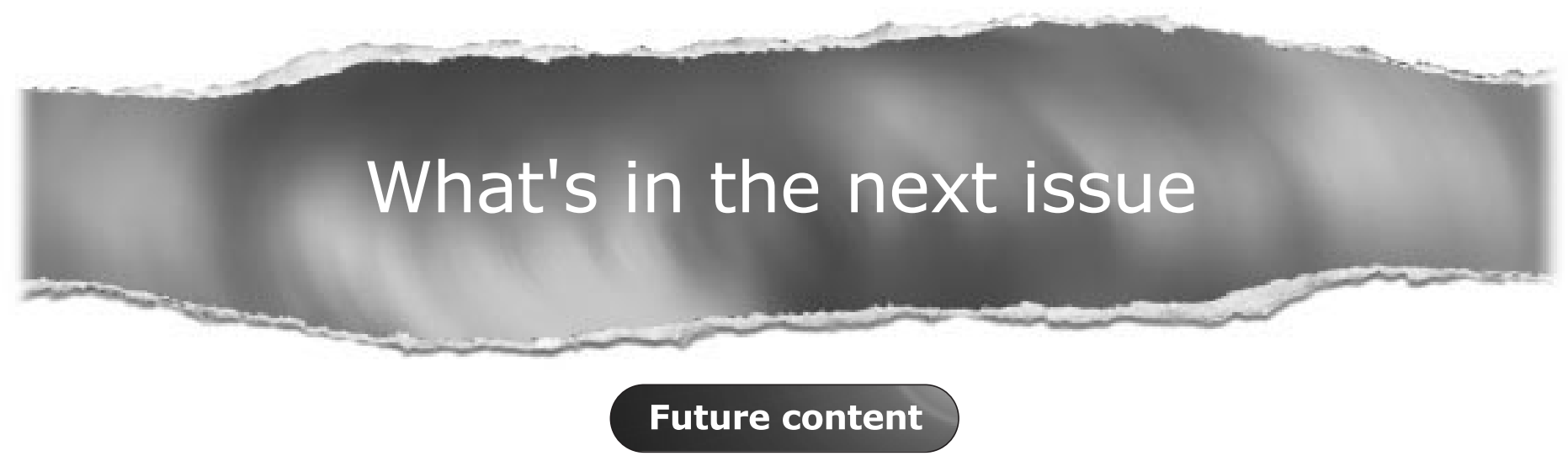

See which articles have just been accepted for publication and preview the table of contents for the next issue a month before it is published

\section{www.jclinpath.com}

